\section{THE : COMPLEMENT FIXATION TEST IN INFLUENZAL PNEUMONIA}

\author{
STUDIES WITH SERUM FROM CONVALESCENT \\ PATIENTS, THE INFLUENZA BACILLUS \\ BEING USED AS ANTIGEN *
}

F. H. RAPOPORT, M.D. (New Haven, Conn.) Lieutenant (Junior Grade) M. C., U. S. N. R. F. CHELSEA, MASS.

These experiments were performed to ascertain whether patients who had recovered from the bronchopneumonia accompanying influenza had developed antibodies that could be demonstrated by the complement fixation test, Bacillus infuenzae being used as antigen.

During the recent epidemic in the First Naval District, attempts were made in this laboratory to demonstrate agglutinins, precipitins or opsonins in the blood of the patients who had the disease. The results so far have been negative with $B$. influenzae. This, indeed, was disappointing, as it was hoped that individuals who had recovered might show some demonstrable changes in the blood. which would indicate the part the immune processes might have played in their recovery.

This expectation was especially strengthened in view of the highly suggestive results obtained by Drs. McGuire and Redden ${ }^{1}$ in their treatment of patients in the active stage of influenzal pneumonia with serum from patients who had recovered from the condition. Hartman, $^{2}$ and Ross and $\mathrm{Hund}^{3}$ have described the use of citrated blood in treating similar cases, and they also report favorable results.

At first, the serums from patients having uncomplicated influenza were tested, but these did not give very satisfactory or encouraging résults. Consequently, the serums from patients who had had influenzal pneumonia were studied.

The object of this series of experiments has been to throw light on these questions:

1. Does the influenza bacillus (found in more than 80 per cent. of the lungs at necropsy in this laborator $\left.{ }^{4}\right)$ give rise to the formation of specific antibodies?

2. Is the complement fixation test of any value in confirming a diagnosis of the bronchopneumonia accompanying influenza?

3 . Can one demonstrate the complement-fixing antibodies in the serums of recovered individuals, which serums have been found valuable in the serotherapy of influenzal pneumonia?

4. Did many strains or only one specific strain of $B$. influenzae become virulent during the epidemic?

\section{ANTIGENS}

Preparation.-Antigens were prepared by various methods, and their respective values were determined

* Owing to lack of space, this article is abbreviated in ThE Journal by the omission of a table giving the results of the tests in detail. The article appears in full in the reprints, a copy of which may be obtained on application to the author.

1. McGuire, L. W., and Redden, W. R.: Treatment of Influenzal Pneumonia by the Use of Convalescent Human Serum, J. A. M. A. 71: 1311 (O'ct. 19) 1918.

2. Hartman, F. W.: New Methods of Blood Transfusion and Serum Therapy, J. A. M. A. 71: 1658 (Nov. 16) 1918 .

3. Ross, C. W., and Fund, E. J.: Transfusion in the Desperate Pneu. monias Complicating Influenza, Preliminary Report on the Successful Use of Total Immune Citrated Blood, J. A. M. A. 71: 1992 (Dec. 14) 1918.

4. Keegan. J. J.: The Prevailing Pandemic of Influenza, J. A. M. A. 71: 1051 (Sept. 28) 1918. by titration. The methods are described in the ordet of antigenic superiority of different preparations:

Method 1: The influenza bacillus was obtained at necropsy from the lung, being grown on blood agar plates. Characteristic colonies were transferred to flasks containing 100 c.c. of glucose broth ( 0.5 per cent.) and 5 drops of sheep's blood previously diluted with broth 200 times (by volume). After three days a good growth usually was obtained. To control the purity of this culture, a loopful of it was streaked out on blood agar, and twenty-four hours later the plate was examined. If this test was satisfactory, 0.3 per cent. tricresol was added to the glucose broth culture and it was again incubated at $37 \mathrm{C}$. for twenty-four hours, when it was tested for sterility. The killed broth culture was centrifuged for twenty minutes at 2,500 revolutions per minute. The supernatant fluid was poured off and the sediment taken up in 40 c.c. of physiologic sodium chlorid solution and washed three times by centrifuging with fresh salt solution ( 0.85 per cent.) each; time. Finally, the washed sediment was taken up in exactly 30 c.c. of saline ( 0.85 per cent.). Sterile beads were added and the suspension shaken for twenty minutes.

Fourteen different antigens were made in this manner, each one being prepared from a culture of influenza bacillus obtained from a different necropsy. Of this number only nine were found to possess good antigenic value.

Method 2: Five antigens made from a mixture of more than one strain of infiuenza bacillus were prepared, in the same way as described in Method 1. Three of these five antigens contained four different strains of the influenza bacillus; one contained six strains, while one had eight strains.

Method 3: These antigens were made as in Method 1, except that after the sediments had been washed, the bacteria were ground up in a sterile mortar and then 30 c.c. of physiologic sodium chlorid solution were added.

Method 4: Sections of lung tissue weighing $2 \mathrm{gm}$. were selected from patients that had died from influenzal bronchopneumonia. These portions of tissue were examined, and when they showed a gram-negative bacillus, morphologically and culturally resembling the Pfeiffer bacillus, 0.3 per cent. tricresol was added and the tissue was ground up in a sterile mortar. Twenty-four hours later, after incubation at $37 \mathrm{C}$., it was tested for sterility. The final volume was made up to 30 c.c. by the addition of salt solution.

Method 5: The influenza bacillus was grown as described in Method 1. The broth culture was centrifuged for twenty minutes at 2,500 -revolutions per minute. The sedintent washed three times with salt solution, and the sediment was then taken up in 30 c.c. of salt solution $(0.85$ per cent.). This suspension was then heated for one-half hour at $56 \mathrm{C}$. It was then tested for sterility by streaking a loopful on bloodagar and incubating for twenty-four hours at $37 \mathrm{C}$. When no growth was evident after twenty-four hours, 0.1 c.c. of 1 per cent. phenol to every cubic centimeter of antigen was added as a preservative.

Method 6: A gram-negative bacillus resembling Pfeiffer's organism, by culture and morphology, was obtained from the bronchial sputum of two patients having influenzal pneumonia. (These patients subsequently recovered from the disease.) The preparation of the antigen was the same as in Method 1.

Method 7: The influenza bacillus was grown on blood agar. A staphylococcus was planted on the plate to cause a more abundant growth. After twenty-four hours, an attempt was made to wash off the influenza colonies; but a staphylococcus contamination could not be avoided by this method. Tricresol, 0.3 per cent., was added, and the final volume was made to 30 c.c. by the addition of physiologic sodium chlorid solution. After twenty-four hours' incubation, the antigen was tested for sterility.

Conclusions from a Comparative Study of These Antigens.-1. The preparations described in Method 1 form the best antigen. 
2. Grinding up the antigen gave only about 20 per. cent. as many positive results as those obtained from the antigen of Method 1.

3 . The polyvalent antigens were of no higher antigenic value than those made from a single strain.

4. Antigens made from bacteria grown on solid mediums (Method 7) were not satisfactory, since the suspensions contained too much blood and it was diffcult to obtain a suspension that did not also contain the staphylococcus. The antigenic value was very low.

5. The antigens obtained from the organism that was found in the bronchial sputum was valueless.

6. Heating apparently destroys, in a large measure, the complement-fixing substance of a bacterial antigen. ${ }^{5}$

7. The organism obtained from the early cases in the epidemic supplied a better antigen for the fixation test than those from the late cases.

8. The antigens do not deteriorate very rapidly. Some of them retained their power to fix complement three months.

\section{TITRATIONS}

The anticomplementary and hemolytic values of each antigen were determined by titration. In finding the anticomplementary dose, varying amounts of the antigen, $0.02,0.05,0.1,0.3,0.5,0.7,0.8,1.3,1.5$ and 2 c.c. of the undiluted antigen, were added to a series of tubes. To each tube, 1 c.c. of $1: 20$ complement was added. The volume of each tube was made to 3 c.c. with physiologic sodium chlorid solution. The tubes were then incubated for one and one-half hours at 37 C. Then 0.5 c.c. of amboceptor containing 2 units and 0.5 c.c. of a 2.5 per cent. suspension of sheep's corpuscles were added. The tubes were reincubated for three-quarters hour, when a reading was made. That tube which first showed inhibition of hemolysis contained the anticomplementary unit of antigen.

Of all the antigens made, only three were found to be anticomplementary. In these instances, the antigenic unit was one quarter of the anticomplementary unit. The many antigens that were not found to be anticomplementary were used undiluted. It was found that usually 0.2 c.c. of the undiluted antigen was the antigenic unit when a definite volume of glucose broth ( 100 c.c.) was used and when the final volume was made to 30 c.c. with salt solution, as described in Method 1.

Each antigen was then titrated against six known positive and six known negative serums before it was used in a series of experiments.

When the antigenic unit was found, this amount of antigen was mixed with 0.5 c.c. of a 2.5 per cent. suspension of sheep's corpuscles, and these were incubated for one-half hour at $37 \mathrm{C}$. This procedure determined whether the antigen was at all hemolytic in the amount representing one unit.

When the foregoing factors were definitely controlled, the antigen could be used in the tests.

Only a brief description of the reagents used is necessary, since the methods of preparation are practically the same as in the Wassermann reaction:

Complement.-Pooled serum of guinea-pig's blood was used. One hour after the blood was obtained, it was centrifuged and the serum was diluted $1: 10$ with physiologic sodium chlarid solution. In preparing the complement for titration, 10 c.c. of physiologic soditum chlorid solution were added to 10 c.c. of the 10 per cent. guinea-pig serum.

5. Perry, M. W., and Kolmer, J. A.: A Study of the Immunizing Properties of Bacterial Vaccines Prepared after Various Methods, J. Immunol. 3 : 258 ( J uly) 1918.
Serum.-The serums were inactivated for twenty minutes at $56 \mathrm{C}$. It was found that heating had also a tendency toward destroying antibodies.

Amboceptor.-The hemolytic amboceptor was the same as that used in this laboratory in doing the Wassermann tests. Briefly, it was obtained by immunizing rabbits with sheep's corpuscles. Two units of amboceptor were used in the fixation tests. The amboceptor was diluted so that 0.5 c.c. contained two units.

Sheep's Cells.-These were obtained by a puncture of the jugular vein of the sheep under sterile precautions. The blood was defibrinated with sterile beads, and then 10 c.c. of the blood were put in a 40 c.c. graduated tube. Physiologic sodium chlorid solution was added to 35 c.c. This corpuscle suspension was centrifuged for fifteen minutes at 2,500 revolutions per minute. These corpuscles were washed three times in this way. After the final washing, the supernatant fluid was poured off and the packed cells were read. A 2.5 per cent. suspension of these washed sheep's cells was made by diluting with physiologic sodium chlorid solution.

Sensitized Corpuscles.-Sensitized corpuscles consist of equal parts of 2.5 per cent. suspension of sheep's corpuscles and diluted amboceptor. This mixture was incubated for one-half hour at 37 C. One c.c. of this mixture was used in the complement fixation test.

Before each series of tests, a preliminary combined titration of complement and amboceptor was made, as shown in Table 1.

TABLE 1.-PRELIMINARY COMBINED TITRATION OF COMPLEMENT AND AMBOCEPTOR

\begin{tabular}{cccc}
\hline \hline & Complement & Amboceptor & Sheep's Cells \\
Tube & $\begin{array}{c}\text { Compilution } \\
\text { 1:20 Units in 0.5 c.c. }\end{array}$ & $2.5 \%$ Suspension \\
& C.c. & C.c. & C.c. \\
1 & 0.5 & 0.5 & 0.5 \\
2 & 0.4 & 0.5 & 0.5 \\
3 & 0.3 & 0.5 & 0.5 \\
4 & 0.2 & 0.5 & 0.5 \\
5 & 1.0 & 0.3 & 0.5 \\
6 & 1.0 & 0.2 & 0.5 \\
\hline
\end{tabular}

To each one of the six tubes in Table 1, physiologic sodium chlorid solution was added to make a volume of 3 c.c. The tubes were incubated at $37 \mathrm{C}$. for onehalf hour. In reading, the least amount of guinea-pig serum causing complete hemolysis determined the size of the unit of complement. If the amboceptor was satisfactory, Tube 5 showed complete hemolysis, while Tube 6 showed moderate hemolysis.

After the combined titration of the complement and amboceptor had been completed, the complement fixation test was thus performed:

Two rows of tubes were set up, as indicated in Table 2. One-tenth c.c. of each of the serums was flowed into one of the tubes in each of the two rows. To each tube in the front row 0.2 c.c. of the antigen and one unit of complement were added. To the rear tubes only one unit of complement was added. The volume of every tube was made to 2 c.c. by the addition of physiologic sodium chlorid solution. Therefore, the front row for each test contained one unit of complement, 0.2 c.c. of antigen and 0.1 c.c. of serum and salt solution, while the respective rear tube contained all of these except the antigen.

These additional controls were made with every series of tests:

(a) Antigen control, containing the unit of complement, the unit of antigen, and physiologic sodium chlorid solution to 2 c.c.

(b) Hemolytic control, containing the unit of complement and physiologic sodium chlorid solution to 2 c.c.

All the tubes were then shaken thoroughly and incubated for one and one-half hours at $37 \mathrm{C}$. Then 1 c.c. 
of sensitized corpuscles was added to each tube and they were reincubated for three quarters of an hour, when a preliminary reading was made. The tubes were put into the refrigerator, and a final reading was made the next morning.

As mentioned above, these tubes were incubated at $37 \mathrm{C}$. for one and one half hours, when 1 c.c. of sensitized corpuscles was added. The tubes were reincubated then for three quarters of an hour and a preliminary reading was made. There should be complete hemolysis in Tubes II, III and IV. If the serum is anticomplementary, there will be inhibition of hemolysis in Tube II, and no reading can be made in Tube I. But if this is not so and there is inhibition of hemolysis in Tube $I$, then this serum is positive, while it is negative when there is hemolysis.

\section{PERFORMANCE OF THE EXPERIMENTS}

As mentioned previously, this series of experiments was performed to determine the occurrence of the complement fixation test on serums from patients who were recovering from influenzal pneumonia, the condition having been determined by physical and clinical signs. To control the specificity of the reaction, a number of individuals were examined who gave no history of the disease during the epidemic and who stated that they were not subject to colds and coughs. Some stress was laid on the last factor, since an ambulatory form of influenza is very probable.

TABLE 2.-SCHEME FOR THE COMPLEMENT FIXATION TEST

\begin{tabular}{|c|c|}
\hline $\begin{array}{c}\text { Tube II } \\
\text { Unknown Serum } \\
\text { 0.1.c.e. of serum (Patient A) } \\
\text { 1 unit of complement } \\
\text { Physiologic sodium chlorid solution } \\
\text { added to } 2 \text { c.c. }\end{array}$ & $\begin{array}{c}\text { Tube IV } \\
\text { Antigen Control } \\
0.2 \text { c.c. of antigen } \\
1 \text { unit of complement } \\
\text { Physiologic sodium chlorid solution } \\
\text { added to } 2 \text { c.c. }\end{array}$ \\
\hline $\begin{array}{c}\text { Tube I } \\
\text { 0.1 c.e. of serum (Patient A) } \\
1 \text { unit of complement }\end{array}$ & $\begin{array}{l}\text { Tube III } \\
\text { Hemolytic Control } \\
1 \text { unit of complement }\end{array}$ \\
\hline $\begin{array}{l}1 \text { unit of antigen ( } 0.2 \text { c.c.) } \\
\text { Physiologic sodium chlorid solution } \\
\text { to } 2 \text { c.c. }\end{array}$ & $\begin{array}{c}\text { Physiologic sodium ehlorid solution } \\
\text { to } 2 \text { c.e. }\end{array}$ \\
\hline
\end{tabular}

It may be questioned whether such serums could be used as satisfactory controls, as it may be that those that did not come down with. the disease had enough antibodies or natural immunity to overcome the virulent organism. This may explain the positive results obtained from some of the controls.

A few serums were tested from patients who still had a high temperature due to the pneumonia, and these are also included in Table 3.

It was endeavored to test blood from patients at different periods of convalescence. Some serums were tested when the temperature had been normal one day, while others were tested when the patients had been convalescent for forty-one days.

When serums were found to be positive, they were later tested at different intervals so as to determine how long the antibodies persisted.

The test and control serums were all negative to the Wassermann reaction, which was performed simultaneously.

MAIN RESULTS FROM THE TESTS

1. Of the 300 control serums tested, twenty-nine, or 9.6 per cent., reacted positively.

2. Of the 315 serums from patients convalescing from influenzal pneumonia, the complement fixation reaction showed 161 positive reactions, 134 negative and twenty anticomplementary reactions. In other words, excluding the anticomplementary serums, 54.5 per cent. of convalescent influenzal pneumonia serums were positive.

3 . Of the 161 positive serums, twenty (12.42 per cent.) were + , thirty-four (21.12 per cent.) were ++ , forty-five $(27.95$ per cent.) were +-++ , and sixtytwo $(38.51$ per cent) were ++++ .

4. Some of the serums were positive one day after the patient had become convalescent. Other serums from patients convalescent forty-one days were positive. Most of the positive reactions were obtained with serums from individuals who were convalescent from three to five days.

5. Some serums that reacted positively three months ago still can fix complements.

TABLE 3.-TESTS IN WHICH WHITE BLOOD COUNTS WERE MADE

\begin{tabular}{|c|c|c|c|c|c|}
\hline \multicolumn{3}{|c|}{ - Above 10,000 per C.Mm. } & \multicolumn{2}{|c|}{ Below 10,000 pe } & \multirow{2}{*}{ C.Mm. } \\
\hline Case & W. B. C. & Result & Case & W. B. C. & \\
\hline 1 & 16,800 & +++ & 12 & 7,400 & Neg. \\
\hline $\mathbf{2}$ & 17,000 & Neg. & 17 & 2,800 & Neg. \\
\hline 4 & 15,000 & Neg. & 20 & 7,400 & $++t$ \\
\hline $\mathbf{5}$ & 13,000 & ++++ & 24 & 4,400 & $+++t$ \\
\hline 6 & 15,200 & ++ & 29 & 7,600 & Neg. \\
\hline 7 & 11,200 & Neg. & 45 & 5,000 & $++t$ \\
\hline 9 & 12,600 & Neg. & 47 & 9,400 & $\begin{array}{l}\text { TT } \\
+++\end{array}$ \\
\hline 14 & 20,000 & ++++ & 55 & 5,400 & Neg. \\
\hline 15 & 14,800 & +++ & 58 & 6,400 & Neg. \\
\hline 16 & 13,000 & Neg. & 57 & 5,800 & Neg. \\
\hline 27 & 10,400 & ++ & 76 & 6,200 & $++t$ \\
\hline 31 & 12,600 & ++++ & 78 & 8,400 & Neg. \\
\hline 41 & 16,000 & $+++t$ & 87 & 6,500 & Neg. \\
\hline 50 & 11,400 & Neg. & 90 & 7,600 & Neg. \\
\hline 58 & 12,200 & Neg. & 92 & 4,200 & + \\
\hline 63 & 14,600 & $++t$ & 97 & 6,200 & $+t$ \\
\hline 65 & 16,000 & ++ & 100 & 5,000 & Neg. \\
\hline 66 & 19,000 & ++++ & 106 & 9,200 & Neg. \\
\hline 72 & 15,200 & $+t$ & 111 & 4,600 & Neg. \\
\hline 73 & 10,200 & + & 287 & 6,600 & Neg. \\
\hline 74 & 28,800 & +++ & 292 & 5,600 & Neg. \\
\hline 80 & 15.200 & & 295 & 3,400 & Neg. \\
\hline 103 & 15,000 & +++ & 303 & 8,400 & Neg. \\
\hline 115 & 16,000 & +++ & 122 & 4,600 & Neg. \\
\hline 124 & 10,200 & ++++ & 130 & 6,200 & Neg. \\
\hline 154 & 12,400 & Neg. & 132 & 4,200 & ++ \\
\hline 158 & 11,200 & ++ & 136 & 8,800 & ++ \\
\hline 166 & 12,400 & ++++ & 142 & 7,800 & Neg. \\
\hline 180 & 13,000 & + & 146 & 4,200 & +1 \\
\hline 197 & 17,400 & Neg. & 152 & 5,200 & Neg. \\
\hline 188 & 15,800 & Neg. & 162 & $\mathbf{5 , 4 0 0}$ & Neg. \\
\hline 192 & 11,800 & ++ & 176 & 8,900 & $\mathrm{~S}$. \\
\hline 194 & 10,400 & Neg. & 178 & 7,000 & $t$ \\
\hline 200 & 13,000 & Neg. & 186 & 9,400 & Neg. \\
\hline 201 & 12,400 & ++++ & 191 & 3,800 & Neg. \\
\hline 208 & 12,400 & Neg. & 198 & 2,800 & Neg. \\
\hline 229 & 12,500 & ++++ & 208 & 5,000 & ++++ \\
\hline 234 & 15,400 & Neg. & 210 & 7,400 & Neg. \\
\hline 238 & 14,600 & $+++t$ & 213 & 4,600 & Neg. \\
\hline 248 & 11,400 & - Neg. & 223 & 5,800 & Neg. \\
\hline 250 & 18,200 & ++++ & 241 & 8,800 & Neg. \\
\hline 258 & 12,600 & +++ & 243 & 7,400 & Neg. \\
\hline 260 & 18,200 & +++ & 249 & 6,400 & Neg. \\
\hline 272 & 18,000 & ++++ & 251 & 5,400 & + \\
\hline 279 & 15,000 & Neg. & 261 & 9,200 & Neg. \\
\hline 283 & 15,200 & Neg. & 274 & 4,400 & + \\
\hline 288 & 17,000 & Neg. & 276 & 5,200 & Neg. \\
\hline 291 & 10,600 & Neg. & 285 & 4,500 & Neg. \\
\hline 299 & 25,000 & $+++\div$ & 286 & 6,200 & Neg. \\
\hline
\end{tabular}

* In this column + indicates hemolysis between 75 and 100 per cent. ++ , hemolysis between 50 and 75 per cent.; +++ , hemolysis between 25

6. The fixation test on many serums made during the time that the patient was still acutely ill or just beginning convalescence were in many cases negative or slightly positive; but when these serums were kept in the refrigerator for from six days to three weeks, many gave strongly positive reactions.

7. White blood counts were not made on all influenzal pneumonia patients admitted, but of those patients on whom one was made and whose serums were tested by the complement fixation test, Table 3 has been made. This table shows that of the forty-nine patients who had a white count above 10,000 , thirty (61.28 per cent.) gave a positive reaction; while of 
the same number having a white count below 10,000 per cubic millimeter, only fifteen ( 30.61 per cent) produced a positive complement fixation test.

\section{COMMENT}

The complement binding factor of serum from influenzal pneumonia patients is probably a very weak one. At first, when two units of complement were used, many more negative results were obtained than when one unit was finally resorted to. Two units probably produced a marked excess of complement for that factor of the serum that causes complement fixation.

The results suggest strongly that the influenza bacillus forms specific antibodies that will fix complement when this organism is used as an antigen.

It is difficult to conclude definitely from these experiments alone whether only one or many strains of the influenza bacillus were active during the epidemic; but this can be stated: Polyvalent antigens did not give more positive results than a monovalent one. If many strains of this organism were present, then one could have expected more positive reactions in these experiments $t h$ a $n$ were obtained from the use of an antigen made from one strain.

Serums that gave positive tests were also found more suitable for the serotherapy of influenzal $p$ n e u monia patients. It seems possible that persons who have not had influenzal pneumonia, but whose serum gives a positive reaction, might be useful as donors.

Since some of the reactions were inconstant, it must be concluded that the complement fixation test in influenzal pneumonia cannot yet be accepted as a diagnostic test; but it may be of some value together with
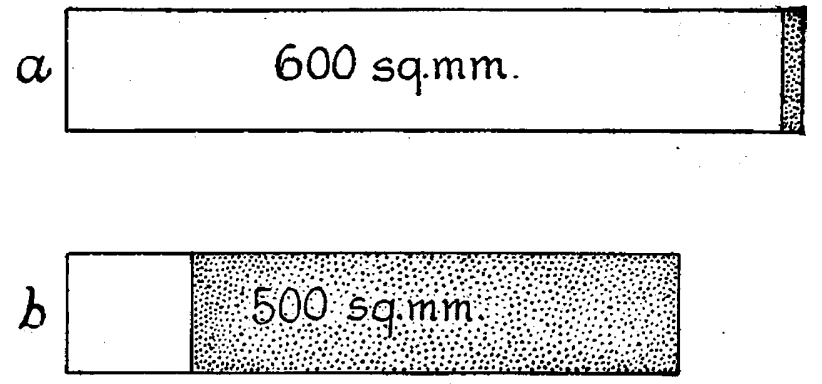

other diagnostic methods in confirming the condition as having been present, at least in more than 50 per cent. of the cases.

This presence of complement fixation would indicate that the influenza bacillus plays an important part in the complicating pneumonia of influenza; but the results furnish no conclusive evidence of a primary etiologic rôle of the influenza bacillus.

\section{SUM MARY}

Experinents were made for the purpose of determining whether specific antibodies existed in the serum of patients who were convalescing from influenzal pneumonia.

The influenza bacillus found in more than 80 per cent. of the necropsies at this laboratory was used as an antigen.

The presence of antibodies was demonstrated by the complement fixation test, for it was found that out of the 300 control serums 9.6 per cent. gave a positive reaction, while of the 295 serums from convalescent influenzal pneumonia patients, 54.5 per cent. had antibodies that fixed complement.

\section{THE TRANSMISSION OF INFECTION THROUGH THE EYE}

\author{
KENNETH F. MAXCY, M.D. \\ Captain M. C., U. S. Army \\ FORT SILL, OKLA.
}

Recently the eye has received little or no attention as a factor in the transmission of acute respiratory infections.. It has been disregarded in planning measures for the prevention of the spread of contagious diseases. This was especially true in the recent epidemic of influenza. No provision has been made in the ward routine of contagious hospitals for the protection of the eye of healthy persons in attendance on the unmasked sick. A brief consideration of the anatomic relations and physiologic facts of the lacrima. tory-nasal mechanism, together with certain simple experiments illustrating its modus operandi, will convince one of the reality and importance of this portal of entry for pathogenic micro-organisms.

It has long been known that large numbers of various organisms, including the pneumococcus, streptococcus, influenza bacillus, and many others, may be recovered from $t h \mathrm{c}$ conjunctival sac, especially if there is obstruction to the overflow of tears. The conjunctival sac is never sterile. The source of contamination has been attributed largely to the impingement on the conjunctiva of the eyeball and the cornea of $\mathrm{dust}$ particles bearing $\mathrm{micro-}$ organisms. It is evident that droplets sprayed from the mouths of other persons during the act of talking, laughing, coughing, sneezing, etc., must be another frequent source. It is now generally accepted that many contagious diseases are transmitted from one person to another through this direct droplet spray. Experimentally it has been shown by Doust and Lyon ${ }^{1}$ that a person infected with a micro-organism ( $B$. prodigiosus) may project this organism from his mouth during ordinary conversation or loud speech to a distance of 4 feet, and that during coughing, infected material from the mouth might be projected at least 10 feet. These observers, together with Haller and Colwell, ${ }^{2}$ proved that this promiscuous spraying of the environment by an infected person could be entirely prevented by the wearing of a properly constructed gauze face mask. There is no experimental proof, however, that the reverse relation holds true, that is, that the noninfected healthy person is entirely protected from droplet spray by the wearing of such a mask. Droplets may not enter the nose or mouth when a face mask is worn, but the conjunctiva remains openly exposed. The relative

1. Doust. B. C., and Lyon, A. B.: Face Masks in Infections of the Respiratory Tract, J. A. M. A. 71. 1216 (Oct. 12) 1918.

2. Haller, D. A., and Colwell, $R$. C.: The Protective Qualities of the Gauze Face Mask, J. A. M. A. 'ं1: 1213 (Oct. 12) 1918. 